\section{ORIGINAL RESEARCH}

A.O. Ortiz

R. Bordia

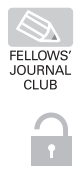

\title{
Injury to the Vertebral Endplate-Disk Complex Associated with Osteoporotic Vertebral Compression Fractures
}

\begin{abstract}
BACKGROUND AND PURPOSE: MR imaging has been used extensively for identification and determination of chronicity of VCF. A major emphasis is placed on identification of edema or fluid clefts within the vertebral body. Little attention is given to other spinal structures such as vertebral endplate, intervertebral disk, posterior elements, and facet joints, spinal ligaments, and paraspinal musculature. Our objective was to assess the incidence of vertebral endplate and adjacent-level intervertebral disk injury as seen on MR imaging studies of patients with acute or subacute painful osteoporotic vertebral compression fractures.
\end{abstract}

MATERIALS AND METHODS: We performed a retrospective review of spine MR imaging examinations in all patients who presented to our institution for subsequent treatment with vertebral augmentation. One hundred six patients had MR imaging studies and reports available for review. A total of 211 acute or subacute vertebral compression fractures were identified in this group of patients. All fracture levels were evaluated for the presence or absence of vertebral endplate and/or associated intervertebral disk injury.

RESULTS: Detailed analysis of the images showed a high incidence of endplate and intervertebral disk injury. Superior vertebral endplate injury (39\%) was more common than inferior endplate injury (12\%), while the combination of both was observed in $29 \%$ of the levels. The pattern of intervertebral disk injury was similar, with injury to the disk above the fracture $(36 \%)$ more common than injury to the disk below the fracture level (15\%). While the official radiology reports correctly identified the vertebral compression fractures, they did not mention or describe the associated vertebral endplate or disk abnormalities.

CONCLUSIONS: Vertebral endplate injury is commonly seen in osteoporotic vertebral compression fractures. Furthermore, this is frequently associated with injury to the adjacent intervertebral disk. These findings are often under-reported but should be described because they may have important implications for symptomatic presentation, patient management, and outcomes.

ABBREVIATIONS: OVCF $=$ osteoporotic vertebral compression fracture

0 VCF of the thoracic and lumbar spine can be a source of disabling back pain. MR imaging of the spine cannot only identify these vertebral compression deformities but also give a relative estimate of fracture acuity by detecting the presence of vertebral body marrow edema, especially on fluid-sensitive sequences such as inversion-recovery and T2-weighted sequences. ${ }^{1}$ Patients with symptomatic acute or subacute osteoporotic vertebral compression fractures are often considered potential candidates for treatment with vertebral augmentation procedures. Procedures such as vertebroplasty and kyphoplasty provide significant pain relief following stabilization of the fractured vertebra. ${ }^{2}$ A major emphasis has been placed on trying to identify edema or fluid clefts within the vertebral body. Scant attention has been given to other spinal structures such as the vertebral endplate, the intervertebral

Received April 1, 2010; accepted after revision May 28.

From the Department of Radiology, Winthrop University Hospital, Mineola, New York

Abstract previously presented at: Annual Symposium of American Society of Spine Radiology, February 19-22, 2009; Lake Buena Vista, Florida. It received the Mentor Award second place.

Please address correspondence to Ritu Bordia, MD, Department of Radiology, Winthrop University Hospital, 259 First St, Mineola, NY 11501; e-mail: rbordia@winthrop.org

Indicates open access to non-subscribers at www.ajnr.org

DOI 10.3174/ajnr.A2223 disk, the posterior elements and facet joints, the spinal ligaments, and the paraspinal musculature. An axial load significant enough to damage the vertebral body could certainly be associated with enough force to cause damage to $\geq 1$ of these aforementioned structures in what is already a compromised spinal column. The purpose of this study was to evaluate 2 other prominent anterior column components of the spinal column, the vertebral endplate and the intervertebral disk. We sought to determine the frequency with which these structures are damaged as evidenced by the same imaging technique that is used to determine acute or subacute vertebral body injury. It is our firm belief that a better understanding of the structural damage that occurs during osteoporotic vertebral collapse will not only result in the improvement of treatment techniques but may also add to our knowledge of other posttreatment challenges such as persistence of back pain symptoms or adjacent-level fractures.

\section{Methods and Materials}

Institutional review board approval was obtained for this study. The MR imaging examinations of the thoracic and/or lumbar spine were reviewed by an experienced neuroradiologist in 106 patients who were evaluated for severe back pain and who subsequently were found to have $\geq 1$ OVCF. The patients ranged from 40 to 94 years of age, with a mean age of 79.4 years. Eighty-eight patients were women, and 


\begin{tabular}{lccccc}
\hline \multicolumn{4}{l}{ Summary of vertebral endplate and disk injury } \\
\hline
\end{tabular}

a Evidenced by abnormal MR imaging findings with respect to the superior and/or inferior vertebral endplate and the disk immediately above and/or below the vertebral compression fracture.

18 were men. Their spine MR imaging examinations were performed on different MR imaging scanners in inpatient and outpatient settings. Some patients underwent spine MR imaging on low-field open magnets at $0.6 \mathrm{~T}$, while most patients $(n=75)$ underwent studies on $1.5 \mathrm{~T}$ units. The sequences that were analyzed included T1-weighted and fast spin-echo T2-weighted sagittal and axial images. One-half of these patients also had sagittal inversion-recovery sequences available for analysis.

A total of 211 acute or subacute vertebral compression fractures, as determined by a combination of clinical presentation and the presence of marrow edema on T2-weighted and/or inversion-recovery sequences, were identified in this patient sample. All of these patients presented within 3 weeks to 3 months of onset of severe back pain that was not responsive to conservative management. Endplate injury was determined by the presence of endplate edema or fluid collection, cortical discontinuity or angulation, or intrusion of disk material into the endplate. This group of patients did not undergo concomitant CT spine imaging. Disk injury was identified by the presence of disk edema or morphologic alteration compared with adjacent levels. These findings were recorded for each fracture level and the adjacent intervertebral disk. They were also subsequently compared with the official written radiology reports of this patient group. All studies were interpreted by board-certified radiologists $(n=21)$, most of whom were subspecialty trained in neuroradiology ${ }^{3}$ or musculoskeletal radiology. ${ }^{4}$

\section{Results}

One hundred two OVCFs were located in the thoracic spine, and 109 OVCFs were located in the lumbar spine. Vertebral endplate injury, as evidenced by the presence of endplate edema or fluid collection, cortical discontinuity or angulation, or intrusion of disk material into the endplate, was observed in 169 of the 211 levels studied (80\%) (Table). Superior endplate injury was more common either as an isolated event in 82 levels (39\%) or in combination with inferior endplate injury in another 62 levels (total superior endplate injury $=68 \%$ ) (Figs 1-3). Inferior endplate injury was observed in 25 levels as an isolated event or in combination with superior endplate injury in 62 levels (total inferior endplate injury $=41 \%$ ) (Fig 4). Disk injury, as evidenced by the presence of disk edema or morphologic alteration (compared with adjacent levels), was observed in 167 of the 211 levels (79\%) studied. The disk above the OVCF was injured in 135 of the 211 levels (64\%), and the disk below the OVCF was injured in 91 of the 211 levels (43\%). There was no evidence of disk hemorrhage, as would be indicated by the presence of T1 signal-intensity hyperintensity, or traumatic disk herniation in this patient population. The combination of disk and endplate injury was observed in 159 of the

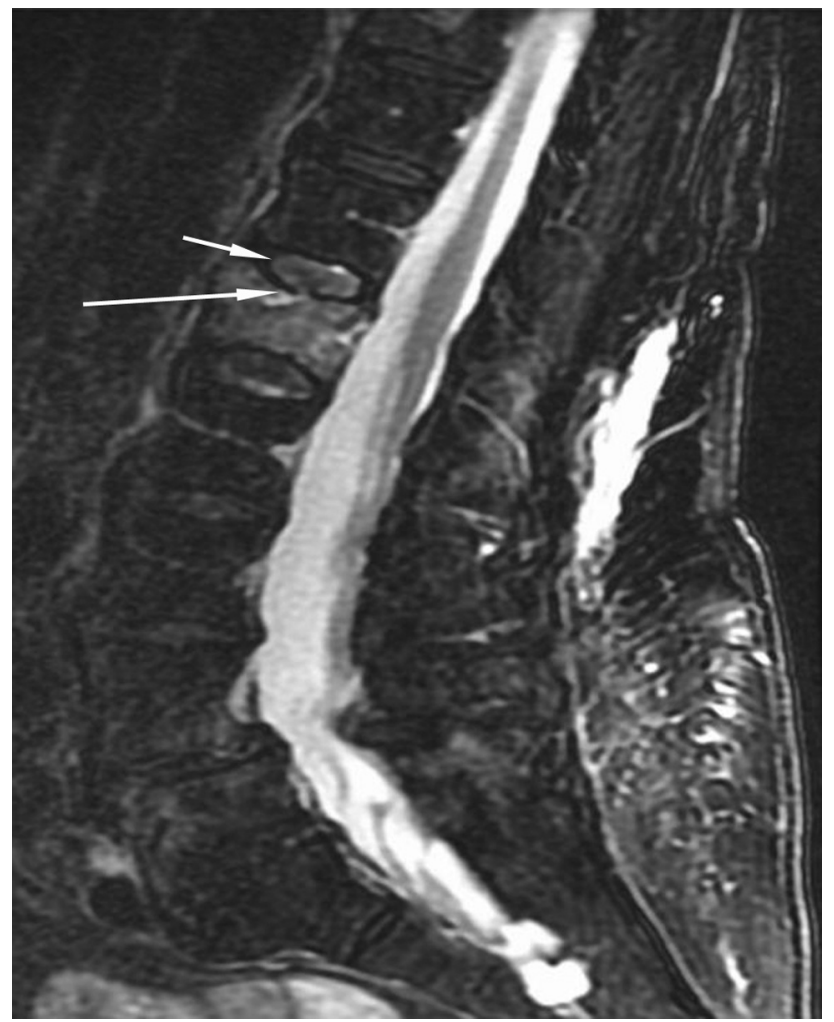

Fig 1. An 88-year-old woman with a 3-week history of severe low back pain. Fatsuppressed T2-weighted sagittal MR image shows diffuse marrow edema within the L1 vertebra and mild height loss consistent with an acute osteoporotic vertebral compression fracture. Focal cortical discontinuity (large arrow) is seen within the superior endplate due to a fracture. This is associated with abnormally increased signal intensity within the T12-L1 intervertebral disk (small arrow), which is consistent with injury to this structure.

211 levels (64\%). Disk injury was not mentioned in any of the official radiology reports in these 106 spine MR imaging studies, whereas vertebral endplate injury was either indirectly or directly mentioned in 30 of the MR imaging examinations (28\%).

\section{Discussion}

The extent of spine injury that occurs during vertebral body collapse in patients with traumatic or spontaneous OVCFs can be significant. A primary focus has historically been placed on the damaged vertebra because the findings of an acute or subacute vertebral compression fracture are readily identifiable with conventional imaging examinations. ${ }^{1}$ Furthermore, current treatment strategies have been aimed at stabilizing these painful vertebral compression fractures with vertebral augmentation techniques such as vertebroplasty and kyphoplasty. $^{2}$ While this paradigm has resulted in a reasonable amount of treatment success, it has not completely addressed nor fully explained many of the clinical concerns and issues that are present in this field of study. Our current study forces us to revisit important basic principles with respect to spine biomechanics. ${ }^{4}$

For the purposes of better understanding our results, it is best to think of the spine as comprising multiple sequentially arranged functional spine units. These spine units include the intervertebral disk that is located between adjacent vertebral bodies. The intervertebral disk not only plays a role in allowing motion between adjacent spinal segments but also is involved 


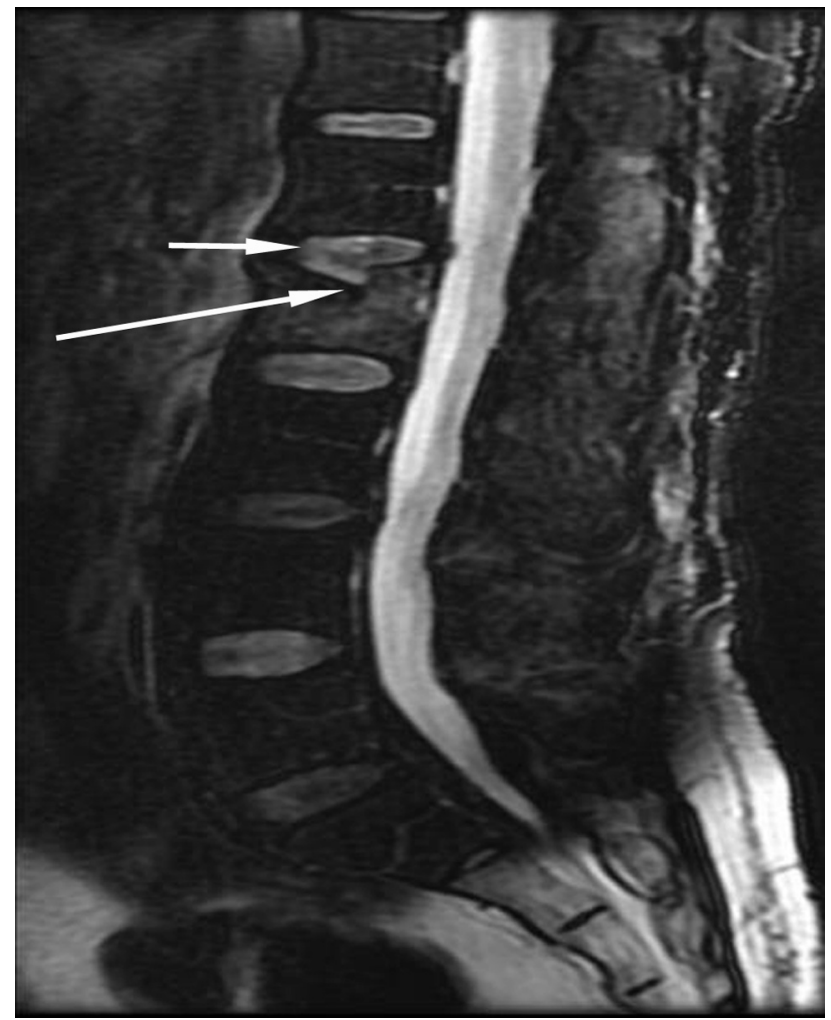

Fig 2. A 62-year-old man with diabetes admitted with a 1-week history of debilitating low back pain. Fat-suppressed T2-weighted sagittal MR image shows diffuse marrow edema within the L2 vertebra. Inferior angulation of a fractured superior endplate (long arrow) is observed. Note the alteration of signal-intensity change and morphology of the injured L1-2 intervertebral disk (small arrow) compared with the neighboring intervertebral disks.

in axial load transfer, especially between the vertebral endplates of these vertebrae. ${ }^{4,5}$ Our results do indicate a high frequency of vertebral endplate injury and disk injury in patients who have painful osteoporotic vertebral compression fractures. The greater incidence of superior endplate and adjacent disk involvement that is observed in this series of patients lends support to a mechanism of axial load transfer as the injury vector in these patients. The axial load is somewhat asymmetric in that it is often associated with flexion and increased stress on the anterior column of the spine. This is the precise location of the vertebral endplate and disk injuries that were observed in our patient population. Most patients in our study were elderly and thus had a higher incidence of degenerative disk changes, particularly within the lumbar spine. A degenerated disk is less capable of attenuating an axial load, and such an axial load tends to be distributed asymmetrically across the vertebral endplate. An injury to the intervertebral disk could further compromise its normal biomechanical properties.

These observations have significant diagnostic implications for the imaging evaluation of patients with osteoporotic vertebral compression fractures. From an imaging standpoint, it is important not only to identify the morphologic alteration and marrow edema that occur with these fractures but also to carefully scrutinize the adjacent intervertebral disks and to note whether there is morphologic and/or abnormal signalintensity change in these structures as well. Furthermore, careful attention should be given to subtle endplate signal-inten-

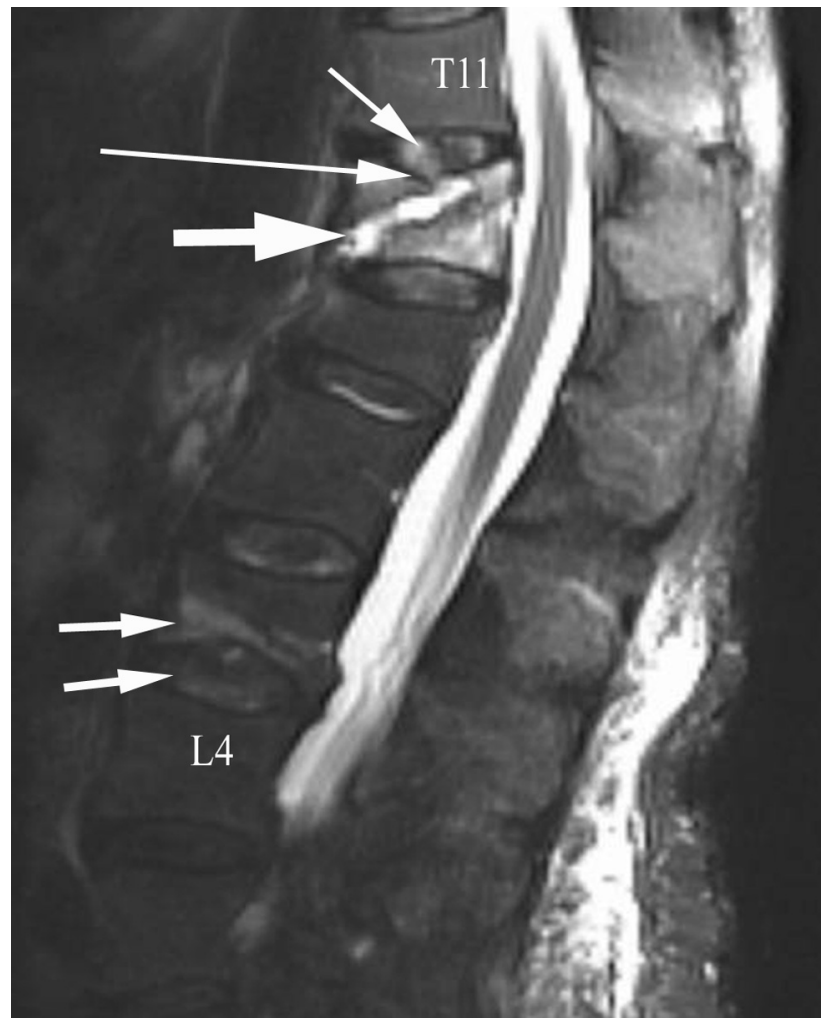

Fig 3. A 79-year-old woman with a 6-week history of low back pain. Fat-suppressed T2-weighted sagittal MR image shows diffuse marrow edema within the T12 vertebra and mild height loss consistent with an acute osteoporotic vertebral compression fracture. A large fluid-filled fracture line (bold arrow) is seen coursing obliquely toward a fractured superior endplate (large arrow). The T11-12 intervertebral disk demonstrates edema (small arrow). An inferior $\mathrm{L} 3$ endplate fracture is associated with $\mathrm{L} 3-4$ intervertebral disk edema (double arrows). A chronic L1 vertebral compression deformity is present.

sity changes in adjacent vertebrae, which may, in actuality, represent additional vertebral fractures in the same patient (Figs 5 and 6). These subtle, or occult, endplate fractures may account for persistent back pain after vertebral augmentation and may then go on to be incorrectly labeled as new adjacentlevel fractures, when they were there all along but were not identified or discussed in the radiology report. These endplate fractures become more apparent on imaging with time and are identified on follow-up evaluation. In those patients in whom adjacent endplates are injured as well as the intervening disk, the initial MR imaging presentation may mimic spine infection. It is not uncommon for patients with this type of disk and endplate injury pattern to be initially referred for biopsy for suspected spine infection (Fig 7). This often causes a treatment delay because vertebral augmentation is contraindicated in patients with suspected spine infection.

The presence of intervertebral disk and endplate injury might serve as a valid explanation for the patient's symptomatic presentation under several clinical scenarios. The intervertebral disk annulus is innervated. ${ }^{6}$ In patients with acute and subacute vertebral compression fractures, motion at the level of the disk-endplate complex during normal flexion activities (such as bending forward to put on socks) or activities that increase axial loading (such as standing or walking) may stimulate the nerve endings in these damaged areas and produce pain. In those patients with chronic painful vertebral compression fractures, the source of pain may be related to 


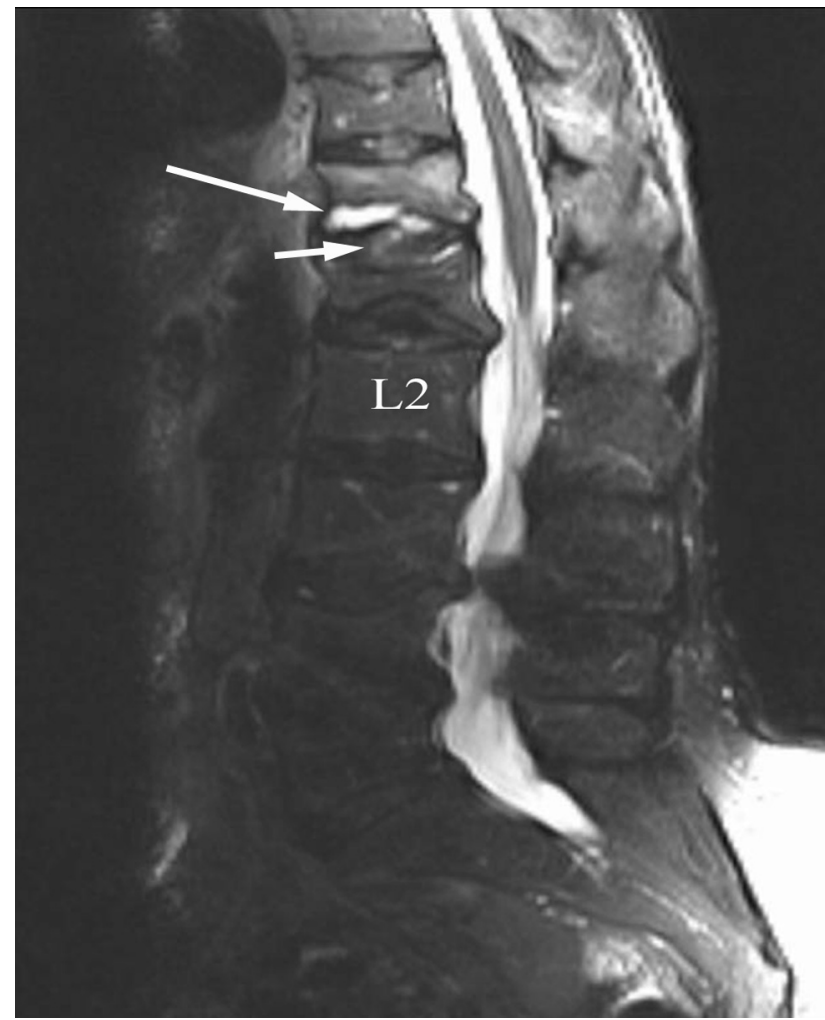

Fig 4. A 78-year-old woman with a 3-week history of low back pain. Fat-suppressed T2-weighted sagittal MR image shows an inferior T12 endplate fracture associated with a fluid-filled cleft (large arrow). Focal edema (small arrow) is noted within the adjacent intervertebral disk. A chronic L1 vertebral compression deformity is present.

instability of the disk-endplate complex. This is not infrequently observed within the thoracic spine, where the constant motion of breathing superimposed on an osteopenic spinal column predisposes to incomplete fracture healing. The latter is associated with hypermobile endplates during fluoroscopic evaluation. Alternatively, axial loading may predispose the disk to move through a damaged vertebral endplate, which in turn may result in chronic pain. In those patients who have been treated with vertebral augmentation but still have vertebrogenic pain, the source of their pain may be related to an incompletely treated vertebral body with failure to stabilize the endplate. ${ }^{7}$

It is our belief that enhanced operator awareness of the increased frequency of endplate injury should alter treatment strategies during vertebral augmentation procedures. A primary objective of vertebral body reconstruction should be to re-establish the structural integrity of the vertebral endplate. This should be accomplished by attempting to appropriately realign the endplate and by sealing any endplate defects that are present. This principle also applies to severe compression deformities with or without small intravertebral clefts. ${ }^{8} \mathrm{Re}-$ pairing an endplate defect prevents disk prolapse into the defect during axial loading, which, in turn, allows the disk to resume its normal biomechanical behavior during load transfer. This prevents an exposure to abnormally high axial loads by the adjacent vertebral body. Furthermore, vertebral endplate repair avoids the leakage of bone cement into the intervertebral disk.

That intradiskal cement leaks occur with a relatively high

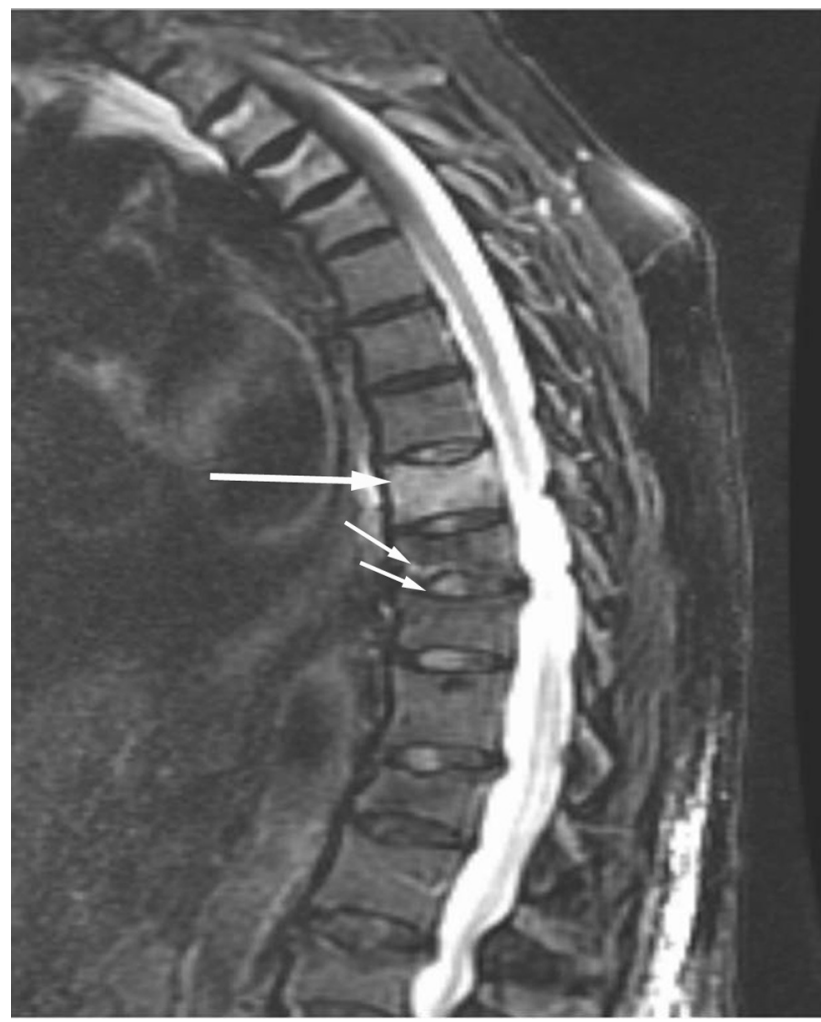

Fig 5. An 84-year-old woman with steroid-related osteoporosis and a 4-week history of severe back pain. Fat-suppressed T2-weighted sagittal MR image shows diffuse marrow edema within the T9 vertebra and mild height loss consistent with an acute osteoporotic vertebral compression fracture (large arrow). In addition, a subtle T10 inferior endplate fracture is associated with adjacent-level disk injury (double arrows). These latter findings were not described in the official radiology report. Yet, on fluoroscopic evaluation, the patient was markedly tender to palpation at the T9 and T10 levels.

frequency in patients with vertebral clefts is consistent with the occurrence of endplate and disk damage. ${ }^{9}$ It has been demonstrated that the presence of intradiskal cement is a significant risk factor for the development of an adjacent-level fracture. ${ }^{10}$ This can be avoided by enhanced operator awareness of the high incidence of endplate damage and by the appropriate use of vertebral body reconstruction techniques by using approaches such as increased-viscosity acrylic bone cement with controlled cement delivery and/or other implants. The ability to perform endplate-to-endplate vertebral augmentation will result in a reconstructed vertebral body that will no longer be a vertebrogenic pain source and will not have adverse biomechanical sequelae for adjacent functional spine units. ${ }^{11}$

The presence of disk and vertebral endplate injury will not only have implications for the application of current treatments and development of new treatments but will also have implications for patient outcomes. A prior vertebral compression fracture in a patient with osteoporosis is a major predictor of subsequent vertebral compression fractures in these patients. ${ }^{3}$ The initial vertebral compression deformity will increase the likelihood of another vertebral compression fracture. Patients with recently treated osteoporotic vertebral compression fractures are also at risk for the development of an adjacent-level fracture. ${ }^{12}$ These tend to occur within the first few months after treatment and several explanations have been proposed for this observation. ${ }^{13-15}$ It is likely that the alteration in disk endplate biomechanics within the previously 


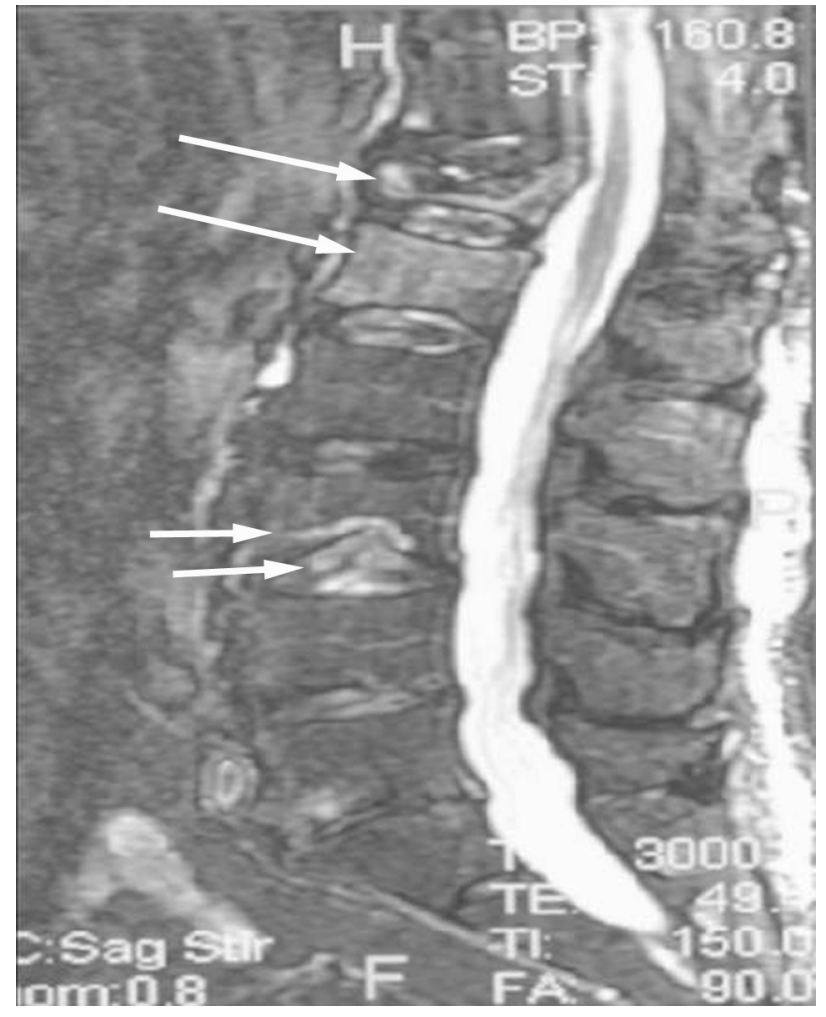

Fig 6. An 82-year-old man with a 3-week history of severe progressive low back pain. Fat-suppressed T2-weighted sagittal MR image shows diffuse marrow edema within the L1 and L2 vertebrae (large arrows), consistent with acute osteoporotic vertebral compression fractures. The superior endplate of $\mathrm{T} 12$ is fractured, and the adjacent disk shows foci of edematous change. An inferior L3 endplate fracture is associated with injury to the adjacent intervertebral disk (double arrows). These latter findings were not reported.

damaged vertebra can have an adverse effect on the adjacent vertebrae, especially if not properly treated with vertebral body reconstruction. Certainly, adjacent-level fractures are often observed in patients who are managed nonsurgically. ${ }^{3}$ It remains to be seen if restoration of the damaged vertebral endplate can lead to a reduced frequency of adjacent-level fractures in patients with osteoporosis.

Our study had several limitations. MR imaging studies of many of our patients were performed at various imaging facilities. The protocol and quality of these studies, therefore, was somewhat variable. Some studies, for example, did not include an inversion-recovery sagittal sequence as part of their protocol. Others were performed on low-field open MR imaging units due to patient claustrophobia issues. These factors made it somewhat challenging to assess the presence and extent of edema in the vertebral body, vertebral endplates, and the intervertebral disk. This could have led to a lowered sensitivity for these findings in these less-than-optimal study situations.

Another limitation relates to the observation that not all patients with osteoporotic vertebral compression fractures are able to undergo an MR imaging study due to implant-related issues. While this latter patient group underwent plain radiography with skeletal scintigraphy or $\mathrm{CT}$ of the spine, the presence or absence of disk pathology could not be evaluated with these latter studies. Because most of our patients were elderly, it can be difficult to distinguish degenerative disk signal-intensity changes and the associated reactive endplate changes from the posttraumatic pathologic changes associated with osteo-

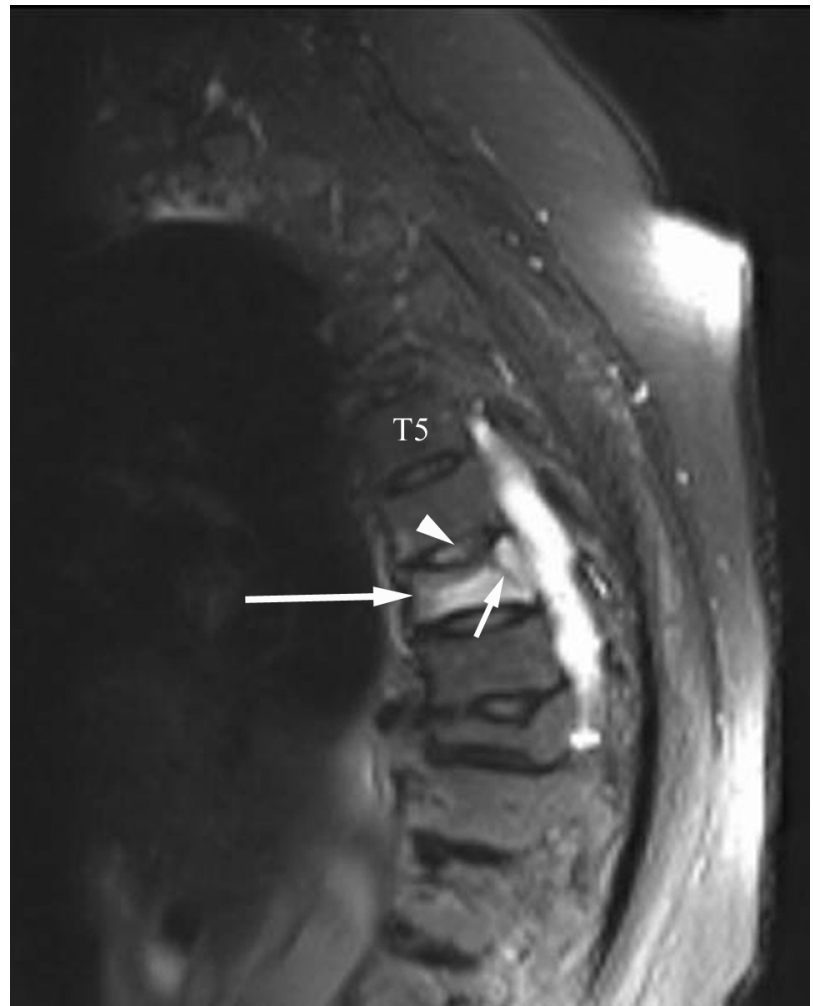

Fig 7. A 90-year-old woman with severe midback pain. Fat-suppressed T2-weighted sagittal MR image (large arrow) shows diffuse marrow edema within the $T 7$ vertebra and mild height loss consistent with an acute osteoporotic vertebral compression fracture. A fracture line (small arrow) extends to the superior endplate. Increased signal intensity is seen within the T6-7 intervertebral disk (arrowhead). This latter abnormality was attributed to possible diskitis in the official radiology report without any consideration given to possible traumatic disk injury in the differential diagnosis.

porotic vertebral compression fractures. Nevertheless, every attempt was made to assess the suspected damaged disk in comparison with the neighboring disk spaces. Follow-up MR imaging studies were not available in this patient population to assess the duration of disk signal-intensity alterations.

In conclusion, vertebral endplate and disk injury are frequently associated with painful osteoporotic vertebral compression fractures as seen on MR images of the thoracic and lumbar spine. The presence of disk and endplate injury should be described in the radiology report because it may have important implications for symptomatic presentation, patient management, and patient outcomes. Additionally, MR imaging examinations should be scrutinized for subtle endplate injuries at levels adjacent to acute vertebral compression fractures because these likely reflect the presence of a second vertebral compression fracture. Treatment strategies should be consistent with the orthopedic principles of fracture reduction, endplate realignment, and fracture stabilization to optimize patient outcomes. Other treatment strategies may include the use of orthotics and physical therapy in appropriately selected patients and osteoporosis management in all patients.

\section{References}

1. Do HM. Magnetic resonance imaging in the evaluation of patients for percutaneous vertebroplasty. Top Magn Reson Imaging 2000;11:235-44

2. Jensen ME, McGraw JK, Cardella JF, et al. Position statement on percutaneous vertebral augmentation: a consensus statement developed by the American 
Society of Interventional and Therapeutic Neuroradiology, Society of Interventional Radiology, American Association of Neurological Surgeons/Congress of Neurological Surgeons, and American Society of Spine Radiology. J Vasc Interv Radiol 2007;18:325-30

3. Lindsay R, Silverman SL, Cooper C, et al. Risk of new vertebral fracture in the year following a fracture. JAMA 2001;285:320-23

4. White AA, Panjabi MM. Clinical Biomechanics of the Spine. 2nd ed. Philadelphia: Lippincott-Raven; 1990:1-81

5. Tzermiadianos M, Renner S, Harvey R, et al. Decreased disc pressure after a vertebral endplate fracture. Is it an additional risk factor for fractures at the adjacent levels? In: Proceedings of the 53rd Annual Meeting of the Orthopaedic Research Society, San Diego, California; February 11-14, 2007: Poster 1028

6. Malinsky J. The ontogenetic development of nerve terminations in the intervertebral discs of man. (Histology of intervertebral discs, 11th communication). Acta Anat (Basel) 1959;38:96-113

7. Lin $\mathrm{CC}$, Wen $\mathrm{SH}$, Chiu $\mathrm{CH}$, et al. The clinical influence of fluid sign in treated vertebral bodies after percutaneous vertebroplasty. Radiology 2009;251:866-72

8. Rad AE, Gray LA, Kallmes DF. Significance and targeting of small, central clefts in severe fractures treated with vertebroplasty. AJNR Am J Neuroradiol 2008;29:1285-87
9. Jung JY, Lee MH, Ahn JM. Leakage of polymethylmethacrylate in percutaneous vertebroplasty: comparison of osteoporotic vertebral compression fractures with and without an intervertebral vacuum cleft. J Comput Assist Tomogr 2006;30:501-06

10. Lin EP, Ekholm S, Hiwatashi A, et al. Vertebroplasty: cement leakage into the disc increases the risk of new fracture of adjacent vertebral body. AJNR Am J Neuroradiol 2004;25:175-80

11. Steens J, Verdonschot N, Aalsma AM, et al. The influence of endplate-to-endplate cement augmentation on vertebral strength and stiffness in vertebroplasty. Spine (Phila Pa 1976) 2007;32:E419-22

12. Uppin AA, Hirsch JA, Centenera LV, et al. Occurrence of new vertebral body fracture after percutaneous vertebroplasty in patients with osteoporosis. $R a$ diology 2003;226:119-24

13. Boger A, Heini $\mathrm{P}$, Windolf $\mathrm{M}$, et al. Adjacent vertebral failure after vertebroplasty: a biomechanical study of low-modulus PMMA cement. Eur Spine J 2007; 16:2118-25

14. Polikeit A, Nolte LP, Ferguson SJ. The effect of cement augmentation on the load transfer in an osteoporotic functional spine unit: finite-element analysis. Spine 2003;28:991-96

15. Baroud G, Nemes J, Heini P, et al. Load shift of the intervertebral disc after a vertebroplasty: a finite-element study. Eur Spine J 2003;12:421-26 